\title{
Emotion Regulation Via Maternal Touch
}

\author{
Matthew J. Hertenstein and Joseph J. Campos \\ Department of Psychology \\ University of California, Berkeley
}

\begin{abstract}
The study of emotion elicitation in the caregiver-infant dyad has focused almost exclusively on the facial and vocal channels, whereas little attention has been given to the contribution of the tactile channel. This study was undertaken to investigate the effects of touch on infants' emotions. During the time that objects were presented to the dyad, mothers provided tactile stimulation to their 12-month-old infants by either (a) tensing their fingers around the infants' abdomen while abruptly inhaling, (b) relaxing their grip around the infants' abdomen, or (c) not providing additional tactile stimulation (control condition). The results revealed that infants in the first condition (a) touched the objects less and waited longer to touch the objects while displaying more negative emotional displays compared to infants in the control condition. However, no apparent differences were found between infants in the second condition (b) and the control condition. The results suggest that infants' emotions may be elicited by specific parameters of touch.
\end{abstract}

Touch has been described as the most fundamental means of contact with the world (Barnett, 1972) and the simplest and most straightforward of all sensory systems (Geldard, 1960). An extensive body of research has been conducted on the importance of touch and infant massage for sensory, cognitive, neurological, and physical development, as well as for pain management and arousal regulation (e.g., Field, 1998). However, the specific role of touch in eliciting and communicating emotions remains largely unexplored (Hertenstein, in press). In this article we present the results of an initial study investigating the processes by which touch elicits and regulates emotion in infants.

The lack of investigation on touch and its relation to emotion is surprising. Touch may be an extraordinarily powerful sensory system for eliciting emotion. The skin contains receptors that directly elicit hedonic values, either because

Requests for reprints should be sent to Matthew J. Hertenstein, University of California, Berkeley, Department of Psychology, 3210 Tolman Hall, Berkeley, CA 94720. E-mail: mat@ socrates.berkeley.edu 
there are portions of the skin that are erogenous or because there are nerve endings that are nociceptive. Furthermore, the information in the environment that affects the skin senses is extremely rich in the perceptual information that elicits emotion. Information reaching the skin senses can vary in rise time, intensity, pressure, temperature, tempo, rhythmicity, directionality (i.e., poking into the skin or moving away from the skin), and shape (the patterning of these various parameters of stimulation). Some of these parameters of environmental stimulation, alone or in concert with others, can elicit the two basic dimensions of all emotion-arousal and, as mentioned, hedonic tone. However, touch may also convey very specific messages like those conveyed by facial displays (Hertenstein, in press; Tronick, 1995). Tomkins (1982) also discussed the idea that various types of stimulation, including touch, may transmit specific emotions as a function of the number and patterning of neural firings per unit of time that result from such stimulation. For example, Tomkins postulated that anger may result if neural firing remains high over an extended period of time, whereas joy may result when neural firing suddenly decreases. In sum, touch is a rich source of information for emotion elicitation and elicits emotion in a variety of ways.

Touch is also a very complex process. Not only do the skin senses encompass many different sensory qualities (including pain, pleasure, pressure, and temperature), even the term touch is complex. Semantically, it can refer to two highly dissociable phenomena, the action of an object on the skin (i.e., touch as contact) and the registration of information by the sensory systems of the skin (what in common usage we call feeling, as in feeling cold or feeling a tickle). Each of these aspects of touch — contact and feeling - may potentially be involved in the elicitation of emotion, although in different ways. The parameters of abruptness, temperature, and so forth listed previously would thus be aspects of contact, whereas stimulation of different locations of the body or triggering the firing of certain nociceptive or pudendal nerves would be aspects of feeling (i.e., sensory processing). It is likely that the functional relations between touch and emotion will be very different depending on which aspect of the global process of touch one is referring to. In this article, contact is used to refer to tactile stimulation, and feel to sensory reception.

\section{EFFECTS OF CONTACT ON AROUSAL, EMOTIONAL EXPRESSION, AND THE ELICITATION OF EMOTION}

To date, the study of contact and emotion has not been systematic, nor has it been broad. In the developmental literature, there has been some work on contact as a regulator of emotions elicited by something else (as opposed to contact eliciting emotions in its own right). For example, maternal contact has been found to soothe 
infants, especially when administered in conjunction with vestibular stimulation (Birns, Blank, \& Bridger, 1966; Korner \& Thoman, 1972). Other studies (e.g., Field, 1983; Koester, Papoušek, \& Papoušek, 1989) have used contact to increase arousal levels and thereby maximize the infant's presumed level of attentiveness or interest.

Other studies have used contact not just to regulate arousal but as a moderating variable in the elicitation of complex emotion blends. Stack and Muir $(1990,1992)$ investigated the role of contact in the still-face situation (Tronick, Als, Adamson, Wise, \& Brazelton, 1978). In this paradigm, the mother assumes an expressionless and stationary facial display directed to her infant while remaining silent-a condition that results in considerable frustration and upset in the child. Stack and Muir (1992) found that when mothers were instructed to contact their 5-month-old infants during this still-face period (maternal contact being left free to vary), their infants displayed significantly less grimacing and more smiling compared to infants whose mothers had not contacted them during the still-face period. The work of Stack and Muir (1992) thus demonstrates that contact plays a significant role in modulating infants' negative emotional displays.

Peláez-Nogueras, Gewirtz, Field, and Cigales (1996) claimed that contact adds to the positive reinforcement value of other forms of reinforcing stimulation. They used the synchronized reinforcement procedure, a contingency-based technique that allows researchers to measure infants' preferences for social stimulation. Peláez-Nogueras et al. (1996) used this procedure to reinforce infant eye contact to the experimenter with two reinforcers: a stimulus compilation that included the face, voice, and contact of an adult versus one that did not include contact. Infants 1.5 to 3.5 months old who received tactile stimulation made more eye contact, emitted more smiles and vocalizations, and spent less time crying and protesting than infants receiving no tactile stimulation. Using the same reinforcement procedure, Peláez-Nogueras et al. (1997) compared the reinforcement effects of tickling and poking to systematic stroking on 2- to 4.5-month-old infants when their mothers directed a slight smile to them. Infants in the latter condition spent a greater amount of time making eye contact with the experimenter, smiled and vocalized more, and cried less.

The work by Stack and Muir $(1990,1992)$ and Peláez-Nogueras et al. (1997; Peláez-Nogueras et al., 1996) demonstrates how contact regulates an already existing action tendency. Contact is used to modulate an extant negative action tendency in the former (Stack \& Muir, 1990, 1992), whereas contact is used to amplify an existing positive action tendency in the latter (Peláez-Nogueras et al., 1997; Peláez-Nogueras et al., 1996). In these studies, the dependent variables focus solely on the expressive component of emotionality.

Contact not only modulates emotion, it can elicit and communicate emotion; moreover, it can do so in many ways. First, the temperature of one's body may elicit emotion in another. For example, cold hands may specify to a perceiver that 
one is in a fearful state (Hatfield, Cacioppo, \& Rapson, 1994), whereas heat may indicate anger in the person doing the touching. Second, haptics, or the dynamics with which one's body moves and impresses on another's skin, is an important component of contact. For instance, fear may be communicated through tremor, anger through abruptness, and love through a complex pattern we call tenderness. Third, the intensity of pressure caused by contact may communicate emotion. Sadness, for example, may be specified by very softly impressing the other's skin, whereas anger may be specified by higher intensities of pressure. Finally, piloerection may elicit the basic emotion of fear in another who feels the person's goosebumps. Although various parameters of contact are listed here individually, emotions are elicited when all the components are orchestrated together in context, thereby resulting in a tactile message to the other that consists of a gestalt. For example, anger may be specified when one contacts the other with (a) an abrupt grasp, (b) a high degree of pressure, (c) a high finger-to-palm ratio of contact, (d) an abrupt release, and (e) skin that feels relatively hot.

In sum, different aspects of contact can elicit and communicate emotion. However, the paucity of studies on how contact elicits emotion prevents any clear inferences about how robustly contact elicits emotion, whether contact is differentially effective at different ages, and how specific the emotions are that contact can elicit.

\section{THIS INVESTIGATION}

This study was meant to be a first step in investigating specific parameters of contact that elicit action tendencies rather than modulate or augment extant ones. Because contact is such a rich modality, it was necessary to select specific parameters of contact to investigate, including haptics and the intensity of pressure, but discard others such as temperature and piloerection that were not easily amenable to experimental control. In addition to investigating the power of contact to affect emotional expressions (as has been done in the previously cited work), we sought to understand how contact affected infants' instrumental behaviors. Finally, like Stack and Muir (1992), we sought to isolate the mother's tactile stimulation from information provided by her face so that the independent effects of contact on the elicitation of emotion could be investigated.

We designed this study to address the following question: Do specific qualities of tactile stimulation provided by the mother in a given context elicit an appropriate emotion or action tendency in the 12-month-old infant (an age at which infants have the developmental and motoric capacities to allow quantification of instrumental behaviors)? We addressed this question by using a paradigm adapted from several social referencing studies (e.g., Klinnert, 1984) in which one of three objects was presented separately to 12 -month-old infants while they sat on their 
mothers' laps. ${ }^{1}$ As the objects were presented, mothers administered contact that was believed to elicit either a negative action tendency or a positive one. We used a pattern of increased stimulation to elicit negative affect and a pattern of decreased stimulation to elicit positive affect.

In the tactile condition designed to elicit a negative action tendency (called the tension increase condition), mothers were instructed to tense their fingers around their infants' abdomen while abruptly inhaling. We predicted that in comparison to a control group, this stimulation would result in more negative and fewer positive emotional displays, as well as less contact with objects, longer duration to contact them, and contacting them with less confidence. The prediction was predicated on the likelihood that this pattern of touch communicated a negative emotion. In contrast, in the tactile condition designed to elicit a positive action tendency (called the tension decrease condition), mothers relaxed their grip around their infants' abdomen while relaxing their posture. We hypothesized that, in comparison to a control group, this stimulation would result in more positive and fewer negative emotional displays as well as more and quicker contact with objects and contacting them with greater confidence. This hypothesis was predicated on the probability that this type of touch would produce a state of relief, which is a type of positive emotion.

\section{METHOD}

\section{Participants}

Thirty-six healthy 12-month-old infants and their mothers were recruited by telephone in the San Francisco Bay Area. Data from 5 other infants were excluded from the analyses because the mother did not follow directions (1), the child had previous experience with one of the objects used in the study (2), or experimenter error (2). The data analyses were conducted on the final sample of 36 infants (half female; mean age $=366.53$ days; $S D=7.84$ days) with 12 in each tactile condition. Infants were typically from middle-class backgrounds. The ethnic composition of the sample was $61 \%$ White, $2.8 \%$ Asian, $5.6 \%$ Hispanic, and 30.6\% classified as other (e.g., mixed race).

\section{Apparatus}

The apparatus in this study included a table, three objects, a curtain, two video recording devices, and a U-shaped manometer. The table $(0.45 \mathrm{~m}$ long $\times 1.22 \mathrm{~m}$ wide $\times$ $0.76 \mathrm{~m}$ high) was equipped with a built-in platform that slid away from or toward the

\footnotetext{
${ }^{1}$ We do not consider this study, strictly speaking, as one on social referencing because the study was not designed to obtain information seeking on the part of the baby.
} 
mother-infant dyad. The experimenter controlled the platform surreptitiously via a handle under the table. The three objects were (a) a black-and-white stuffed cow (25 cm long, $16.5 \mathrm{~cm}$ high, and $12.5 \mathrm{~cm}$ wide) that made a "moo" noise, (b) a stuffed white rabbit ( $18 \mathrm{~cm}$ long, $18.5 \mathrm{~cm}$ high, and $11.5 \mathrm{~cm}$ wide) making a squeaking noise, and (c) a stuffed representation of a pink nose with dangling legs and feet $(14 \mathrm{~cm}$ long, $31.5 \mathrm{~cm}$ high, and $13.5 \mathrm{~cm}$ wide). These objects were chosen because they elicited neither immediate positive nor negative affective displays from infants in an informal pilot test. A black curtain was hung $45 \mathrm{~cm}$ from the edge of the table. Two video cameras were used: One camera recorded the face and upper torso of the infant, and the other camera recorded the dyad from the side. Only the lens of camera one was visible to the dyad, though minimally, through an aperture cut in the curtain. The output of the two cameras was fed into two VCRs for subsequent data analysis. Separate and asynchronous time-date generators were used to superimpose a stopwatch time to the hundredth of a second on each of the video recordings. During the training session, a U-shaped manometer with a black inflatable ball (circumference $=80 \mathrm{~cm}$ ) attached via an air hose was used to help standardize the amount of pressure that the mothers would exert on their infants during experimental trials. When the hose was squeezed, a column of dyed red water rose in the hose, thereby allowing mothers to gauge the pressure with which they grasped the ball.

\section{Test Setting}

The infant-mother dyad was seated at one end of a carpeted room $(7.75 \mathrm{~m} \times 3.49$ $\mathrm{m})$. Experimenter 1 communicated with the mother from behind the dyad, and Experimenter 2 sat behind the curtain to operate the video recording equipment and control the platform presenting the objects to the infant. During the testing session, all communication ceased between experimenters as well as with the dyad. The infant sat on his or her mother's lap and the mother held the infant's torso bilaterally. The dyad sat directly in front of and facing the table and curtain; thus, the infant could not see either experimenter. The objects emerged (via the platform in the table) from behind the curtain.

\section{Design}

The infant-mother dyads were randomly assigned to one of three tactile conditions: (a) tension increase, (b) tension decrease, or (c) a control condition in which the infants received no tactile message. Three objects were presented to each dyad. The objects were presented in a counterbalanced order across conditions constituting three trials for each dyad. Thus, the study was conceptualized as a $3 \times 3$ (Tactile Condition $\times$ Trial) mixed design with repeated measures on trial. 


\section{Training}

All mothers were trained first to hold their infants in the neutral position. Subsequently, mothers were trained for their respective tactile conditions. Figure 1 illustrates the position in which infants were held by the mothers during each trial.

Neutral position. Using a rag doll as a model of an infant, the mother was trained to place her infant on her lap with the infant's back in complete contact with her abdomen. In addition, she was trained to hold her infant bilaterally around the torso so her fingers from both hands interlocked and her thumbs were flat against the infant's abdomen. The force with which she held the infant was equally distributed across her hands. Prior to testing, the U-shaped manometer was used to help standardize the amount of force with which the mothers exerted contact in the neutral position. The mothers exerted pressure on the inflatable ball to raise the column of water in the instrument $3 \mathrm{~cm}$. The mother held the infant in this position at the beginning of each trial.

Tension decrease condition. This condition was designed to elicit positive emotion and to provide such information contingent with the appearance of the object. The mother was trained to loosen her palmar grip around the infant's torso while relaxing her posture. The mother touched the infant throughout the trial. The mother practiced on the U-shaped manometer until she was capable of reliably reproducing a lower intensity of pressure than that of the neutral condition (i.e., $2 \mathrm{~cm}$ lower than the neutral water level). She practiced keeping the intensity with which she touched the inflatable ball equal across the palmar surface of her hands.

FIGURE 1 Illustration of mother holding infant in the neutral position.

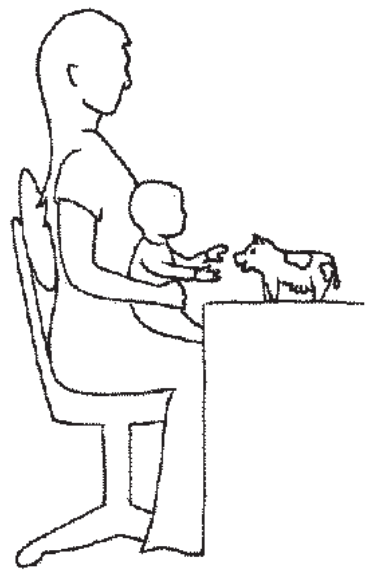


Tension increase condition. This condition was designed to elicit negative emotion and to provide the tactile information contingent with the appearance of the object. Using the rag doll as a training device, the mother practiced rapidly inhaling and tightening her grip around the infant's torso with her fingers. These manipulations were chosen for purposes of ecological validity. Each mother was trained to inhale deeply and rapidly and to hold her breath for approximately $1.5 \mathrm{sec}$. Special care was taken to assure that mothers inhaled inaudibly. Regarding the mother's grip, she practiced on the $\mathrm{U}$-shaped manometer until she was capable of reliably reproducing a pressure intensity higher than the neutral pressure (i.e., $8.5 \mathrm{~cm}$ higher than the neutral water level). In addition, she practiced on the manometer using her fingers, rather than her palms, to increase the force that she would exert on the infant in the trials. The mother was trained to allow $1.5 \mathrm{sec}$ to elapse from the time she began the tactile manipulation to the time that she returned to the neutral position. Care was taken to ensure that the tension increase resulted in no displacement of the infant's body or arms by the mother's action yet permitted the infant to move arms and body freely. Thus, the infant had full access to the objects and was not constrained.

Control condition. In this condition, the mother was trained to remain in the neutral position for the duration of the procedure.

\section{Manipulation Check}

Two manipulation checks were conducted. In the first manipulation check the experimenter listened intently to be sure that mother's inhalation in the tension increase condition was inaudible. All mothers conformed with instructions according to the check. In the second manipulation check, an assistant, blind to the research design and hypotheses, rated from the videotape whether the tactile message given to the infant was in the tension decrease, tension increase, or control condition. The assistant was instructed to attend to the mother's grip around the infant as well as her breathing. Each trial was rated for every participant resulting in 108 ratings ( 3 trials per participant). When compared to the actual conditions to which the participants were assigned, 107 of the 108 ratings were in agreement. The trial in which the assistant did not provide the correct rating was included in the final analysis as originally assigned, not as coded.

\section{Procedure}

Each mother-infant dyad was tested separately. On arriving for the experiment, a female research assistant invited the infant to play with objects on the floor of the laboratory. While the infant played with the research assistant, the experimenter 
trained the mother to hold her infant in the neutral position and as required by the experimental condition to which the dyad was randomly assigned. Following the introductory and training phase that lasted approximately 15-20 min, the mother was instructed to hold her infant in the neutral position. After the infant was in an attentive state and faced forward, the first trial commenced. A hidden research assistant operated the platform in the table to present one of the three objects to the dyad at a rate of approximately $17.9 \mathrm{~cm} / \mathrm{sec}$. From the infant's perspective, the objects emerged from behind the curtain and stopped $7.5 \mathrm{~cm}$ from the edge of the table. On seeing the object, the mother performed the requisite tactile manipulation (tension increase, tension decrease, or no tactile manipulation). Subsequently, the infant had $45 \mathrm{sec}$ to manipulate the object if he or she wished. During this period, the mother allowed the infant to interact with the object. Because the focus of this study was contact, mothers were instructed to maintain a neutral face throughout the procedure just in case the infant looked back at them. However, infants looked at the mothers very infrequently because of the positioning of their bodies toward the objects for the duration of the trial. After the 45 -sec period, the mother placed the object on the moving platform, and the experimenter retracted the object. A 45-sec trial duration was chosen so as not to induce excessive fussiness in the infant. The same procedure was used for the subsequent two trials. Between trials, mothers were allowed to hold their infants or to allow their infants to play on the floor beside them. Approximately 2 min elapsed between trials for each infant. The procedure was designed to be comfortable for both the mother and the infant.

\section{Coding of Infant Behaviors}

Six coders, all of whom were naive to the research questions, hypotheses, and overall research design, coded the infants' hedonic tone, latency to reach toward the object, duration of touching the object, and duration of touching the object with his or her whole hand. ${ }^{2}$ These dependent variables were chosen because they have been used successfully in other studies of the behavior regulatory consequences of emotion (e.g., Camras \& Sachs, 1991; Hirshberg \& Svejda, 1990; Mumme, Fernald, \& Herrera, 1996). For each condition, two assistants coded all the infants in that condition. This coding procedure was crucial to keep the coders naive to the hypotheses of the study. (As the manipulation check demonstrated, the information visible on the video was sufficient for a coder to identify what was a tension decrease and what was a tension increase condition.)

\footnotetext{
${ }^{2}$ The arm withdrawal of the infants from the object as well as the duration of time that the infants contacted the objects with their fingertips were coded. However, these variables possessed inadequate interrater reliability; thus, they were not included in the analysis.
} 
For all dependent variables except hedonic tone, the two coders worked independently, and their ratings were subjected to Pearson product-moment correlations to determine intercoder reliability. For hedonic tone, the two coders for each condition worked together to arrive at a single code per participant per scoring epoch. Subsequently, interrater reliability was determined by correlating (Pearson product-moment) these codes with those of an independent coder (Matthew Hertenstein), who coded one third of the data.

Using the time-date generator stopwatch display on the videotape, the coders scored $40 \mathrm{sec}$ of data for each trial. For all dependent variables, coding of the trial began when the object was approximately $11.75 \mathrm{~cm}$ from the edge of the table. The object traveled an additional $4.25 \mathrm{~cm}$ before stopping in front of the infant.

Hedonic tone. The general hedonic valence of the infant was coded, not displays of discrete emotions. Judgments were based exclusively on facial and vocal displays of emotion. The instrumental behaviors of the infant were masked on the monitor by placing an opaque cover over the neck and torso of the image of the infants so as not to influence judges' ratings. The highest affective display rating that occurred within each of four 10-sec periods was scored (only the first two of the four ratings were subsequently analyzed, due to lack of variation in affective responding past the 20 -sec mark). In addition, ratings were given for the second preceding the trial. A score of 0 indicated that the infant appeared neutral during the scoring epoch. For the Positive Expressive scale, a 1 was given for a weak smile, operationally defined as slight upturning of the mouth while the mouth was closed and was accompanied by no cheek elevation. A 2 was given for more intense smiling, operationally defined as a smile with either an open mouth or elevation of the cheek. A 3 was scored if any of the criteria stipulated in a score of 2 were met and a positive vocalization (cooing, gurgling, etc.) was present concurrently. For the Negative Expressive scale, a 1 was given when the infant frowned, a 2 was given when the infant frowned in conjunction with his or her head turning away from the object or the corners of the mouth were pulled back, and a 3 was given if any of the criteria stipulated in a score of 2 were met and a negative vocalization (grunting, crying, etc.) was present concurrently. Interrater reliability was $r>.90$ for the Positive Expressive scale and $r>.91$ for the Negative Expressive scale.

Latency to contact. The time from which the trial began to the time at which the infant first made contact with the object was coded. Each infant received one latency score for each trial. Interrater agreement was $r>.92$ for all coder groups.

Duration of contact. The total time that the infant contacted the objects was coded. Interrater agreement was $r>.87$ for all coder groups. 
Percentage of whole-hand contact. The percentage of time that the infant contacted the objects with his or her whole hand was coded as an index of the confidence with which the infant touched the objects. This was coded when one or both of the infant's entire palms touched the object or when all four fingers, irrespective of the thumb, contacted the object. The percentage was calculated by dividing the duration of time that the infant touched the object with the whole hand by the duration of total time that the infant contacted the object. Interrater agreement was $r>$ .83 for all coder groups.

\section{Data Analytic Strategy}

Each of the five dependent variables in this study was subjected to the same data analytic strategy. Each was analyzed by a $3 \times 3$ (Tactile Condition $\times$ Trial) analysis of variance (ANOVA) with repeated measures on the last factor. To test our hypotheses directly, the two degrees of freedom for tactile condition were decomposed into two planned comparisons with a one-tailed test on the Tactile factor. ${ }^{3}$ The first contrast compared the tension increase condition to the control condition, and the second compared the tension decrease condition to the control condition. Similarly, the two degrees of freedom for trial were decomposed into a linear and quadratic term.

\section{Preliminary Analyses}

To assure adequacy of random assignment to conditions, one-way ANOVAs were performed with mother's age and income level as dependent variables. No differences were found between conditions regarding these two variables (all $p \mathrm{~s}>.05$ ). Univariate ANOVAs on each dependent measure were conducted to test for interactions between the tactile conditions and sex as well as the objects (rabbit, cow, nose) used in the experiment. No analysis yielded a significant interaction for either sex or object (all $p \mathrm{~s}>.05$ ). Also, univariate ANOVAs were conducted to test for differences between tactile conditions on judges' pretrial ratings of emotionality on both the positive and negative scales. There were no significant main effects or interactions (all $p \mathrm{~s}>.05)$.

Time window. On review of the data, only the first $20 \mathrm{sec}$ of each trial were analyzed. A number of reasons led to this decision. Researchers have found that in

\footnotetext{
${ }^{3}$ As suggested by Keppel (1991), no corrections were used for the contrasts because the comparisons were conducted to test directly hypothesized relations between the tactile conditions. Also, we believe that one-tailed tests were justified because the alternate hypotheses were directional (see Repacholi, 1998 , for a similar view).
} 
paradigms similar to the one used in this study the situation often becomes less ambiguous over the course of a given trial and that the strength of the effect of the mothers' signals may lessen because mothers did nothing after applying the tactile stimulation (Mumme et al., 1996). Because of these findings and the fussiness of many infants over the trial, we decided that the appropriate unit of analysis was a 20-sec window of time. Others (e.g., Mumme et al., 1996) have also reduced the window of time for analysis for similar reasons.

\section{RESULTS}

All the means and standard deviations are presented for each tactile condition in Table 1. Eta-squared $\left(\eta^{2}\right)$ statistics refer to the effect sizes between each of the tactile conditions and the control condition. An $\eta^{2}$ of .01 is considered to be a small effect size, an $\eta^{2}$ of .06 is a medium effect size, and an $\eta^{2}$ of .14 or larger is a large effect size (Clark-Carter, 1997). Variability was comparable in all groups. The marginal means and standard deviations for the Trial factor as well as the eta-squared values for the linear and quadratic terms are displayed for each dependent variable in Table 2. The univariate analyses are reported for each dependent variable in the following sections. For sake of brevity, inferential statistics for interactions are not reported because they are all statistically not significant at the $p>.05$ level (all $\eta^{2}<$ $.05)$. In addition, the linear and quadratic terms were not reported unless they were significant at the $p<.05$ level.

TABLE 1

Means and Standard Deviations by Tactile Condition for Expressive and Instrumental Behaviors

Tactile Condition

\begin{tabular}{|c|c|c|c|c|c|c|c|c|}
\hline \multirow[b]{2}{*}{ Behavior } & \multicolumn{2}{|c|}{$\begin{array}{c}\text { Tension } \\
\text { Decrease }\end{array}$} & \multicolumn{2}{|c|}{$\begin{array}{l}\text { Tension } \\
\text { Increase }\end{array}$} & \multicolumn{2}{|c|}{ Control } & \multirow[b]{2}{*}{$\eta^{2 a}$} & \multirow[b]{2}{*}{$\eta^{2 b}$} \\
\hline & $M$ & $S D$ & $M$ & $S D$ & $M$ & $S D$ & & \\
\hline Negative Expressive scale ${ }^{c}$ & 0.25 & 0.16 & $1.38 * *$ & 0.26 & 0.35 & 0.11 & .39 & .01 \\
\hline Positive Expressive scale ${ }^{c}$ & 1.32 & 0.26 & 0.72 & 0.23 & 0.94 & 0.20 & .03 & .06 \\
\hline Latency to contact ${ }^{\mathrm{d}}$ & 7.69 & 1.60 & $10.44 *$ & 1.99 & 6.09 & 1.44 & .14 & .03 \\
\hline Duration of contact ${ }^{\mathrm{d}}$ & 6.95 & 1.17 & $5.02 *$ & 1.17 & 9.01 & 1.35 & .19 & .06 \\
\hline Whole-hand contact ${ }^{\mathrm{e}}$ & 49.41 & 9.36 & $41.33 *$ & 7.46 & 58.71 & 7.13 & .12 & .03 \\
\hline
\end{tabular}

Note. $\quad n=12$. Eta squared $\left(\eta^{2}\right)$ values refer to effect sizes for planned comparisons.

${ }^{\mathrm{a}}$ Tension increase-control comparison. ${ }^{\mathrm{b}}$ Tension decrease-control comparison. ${ }^{\mathrm{c}}$ Values on a scale ranging

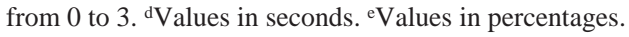

$* p<.05 . * * p<.01$. 
TABLE 2

Means and Standard Deviations by Trial for Expressive and Instrumental Behaviors

\begin{tabular}{|c|c|c|c|c|c|c|c|c|}
\hline \multirow[b]{3}{*}{ Behavior } & \multicolumn{6}{|c|}{ Trial } & \multirow[b]{3}{*}{$\eta^{2 a}$} & \multirow[b]{3}{*}{$\eta^{2 l}$} \\
\hline & \multicolumn{2}{|c|}{1} & \multicolumn{2}{|c|}{2} & \multicolumn{2}{|c|}{3} & & \\
\hline & $M$ & $S D$ & $M$ & $S D$ & $M$ & $S D$ & & \\
\hline Negative Expressive scale & 0.46 & 0.11 & 0.65 & 0.15 & 0.86 & 0.14 & .15 & .00 \\
\hline Positive Expressive scale ${ }^{c}$ & 1.00 & 0.15 & 0.94 & 0.17 & 1.04 & 0.17 & .00 & .01 \\
\hline Latency to contact ${ }^{\mathrm{d}}$ & 8.68 & 1.25 & 7.43 & 1.25 & 8.11 & 1.26 & .00 & .02 \\
\hline Duration of contact ${ }^{\mathrm{d}}$ & 6.58 & 1.07 & 7.00 & 0.88 & 7.40 & 0.93 & .01 & .00 \\
\hline Whole-hand contact ${ }^{\mathrm{e}}$ & 57.05 & 6.53 & 45.79 & 6.71 & 46.61 & 6.67 & .05 & .02 \\
\hline
\end{tabular}

Note. $n=36$. Eta squared $\left(\eta^{2}\right)$ values refer to effect sizes for the linear and quadratic terms.

${ }^{a}$ Linear term. ${ }^{b}$ Quadratic term. ${ }^{c}$ Values on a scale ranging from 0 to $3 .{ }^{d}$ Values in seconds. ${ }^{e}$ Values in percentages.

\section{Negative Expressive Scale}

As predicted, infants in the tension increase tactile condition exhibited more negative emotional displays than those in the control condition (large effect size). However, there were no group differences found between the tension decrease condition and the control condition. The linear term for the Trial factor indicated that infants progressively emitted more negative emotional displays with each trial, $p<.03, \eta^{2}$ $=.15$. The contrast comparing the tension increase condition and the control condition yielded main effects for the tactile condition, $p<.01, \eta^{2}=.39$, and trial, $p<.05$, $\eta^{2}=.13$. The contrast comparing the tension decrease condition and the control condition yielded no effects for the Tactile and Trial factors, all $p s>.05$, all $\eta^{2}<.08$.

\section{Positive Expressive Scale}

Contrary to our prediction, the analyses indicate that the tactile condition to which infants were assigned did not affect their positive emotional displays. The two planned comparisons yielded no main effects for the tactile condition or for trial, all $p$ s $>.05$, all $\eta^{2}<.06$.

\section{Latency to Contact}

As hypothesized, infants in the tension increase condition waited to contact the toy longer than infants in the control condition (large effect size). In fact, infants 
in the former condition waited over $4 \mathrm{sec}$ longer to contact the toys, on average, than infants in the latter condition. However, infants in the tension decrease condition did not differ statistically from infants in the control condition on this dependent variable. The contrast comparing the tension increase condition and the control condition yielded a main effect for tactile condition, $p<.04, \eta^{2}=.14$, but not for trial, $p>.05, \eta^{2}=.03$. The contrast comparing the tension decrease condition and the control condition was statistically not significant for both factors, all $p$ s $>.05$, all $\eta^{2}<.03$.

\section{Whole-Hand Contact}

As predicted, infants in the tension increase condition touched the objects with their whole hands a lower proportion of time than did infants in the control condition (medium effect size). Contrary to our prediction, however, infants in the tension decrease condition did not differ from those in the control condition. The contrast comparing the tension increase condition and the control condition yielded a main effect for the Tactile factor, $p=.05, \eta^{2}=.12$, but not for trial, $p>.05, \eta^{2}=.09$. The contrast comparing the tension decrease and control conditions yielded no significant findings, all $p s>.05$, all $\eta^{2}<.03$.

\section{Duration of Contact}

As predicted, infants in the tension increase condition touched the toys less than infants in the control condition (large effect size). The difference between these groups was robust in that infants in the former condition touched the objects almost $4 \mathrm{sec}$ less, on average, than infants in the latter condition. However, infants in the tension decrease condition did not differ from those in the control condition. The contrast comparing the tension increase condition and the control condition yielded a main effect for tactile condition, $p<.02, \eta^{2}=.19$, but not for trial, $p>.05, \eta^{2}=.05$. The contrast comparing the tension decrease condition and the control condition was statistically not significant for both factors, all $p \mathrm{~s}$ $>.05$, all $\eta^{2}<.07$.

In sum, tension increase stimulation resulted in longer latency and shorter duration of contacting the objects and elicited more negative emotional displays compared to the control condition. In addition, infants in the control condition contacted the objects with their whole hands more than infants in the tension increase condition. Infants' negative expressive displays increased across the three trials. In contrast to the tension increase condition, the tension decrease stimulation affected neither instrumental nor expressive behaviors relative to the comparison group. 


\section{DISCUSSION}

This study provides evidence that specific qualities of touch robustly elicit negative expressions and action tendencies. However, the particular tactile manipulation thought to elicit positive emotions was ineffective in the context of this study. These results accord with the majority of studies designed to investigate the behavior regulatory effects of emotions indicating that negative emotions may be more readily elicited and regulate behavior more robustly than positive emotions (Boccia \& Campos, 1989; Feinman \& Lewis, 1984; Mumme et al., 1996; Svejda, 1981).

What exactly do these findings reveal about the relevance of tactile stimulation for the elicitation of emotion? First, it appears that the array of tactile stimulation in the tension increase condition not only affected the child's emotional expressions in the predicted manner, but it did so almost immediately. This suggests that some aspects of tactile stimulation are peremptory. Second, there was evidence that the increasing tension condition had a cumulative effect over trials, as evidenced by infants' expressive displays. Third, infants in the tension increase condition touched the objects with their whole hands (an index of confidence) less than infants in the control group. Fourth, the tactile stimulation in the tension increase condition regulated infants' behaviors across three different objects, indicating that the effect generalized across objects. In sum, the tension increase condition inhibited the child's behavior and was increasingly effective over time and trials.

\section{Tension Decrease and Emotion}

Why, in contrast to the tension increase condition, did the tension decrease condition have no apparent effect on infants' emotions? There are a number of explanations that may account for this finding. First, the wrong positive emotion may have been manipulated. Positive emotions are typically considered to be limited to a single type (e.g., joy), but in fact there are many kinds of positive emotion-contentment, exhilaration, triumph, glee, and relief, to list but a few. In this experiment, the tension reduction condition provided information more relevant to eliciting relief than other positive emotions. However, in a nonthreatening context such as the one in this experiment, there was nothing to be relieved about. If the context had been slightly frightening to the child, relief elicited by tension decrease may have resulted in the child's behavior being significantly encouraged.

There is a second explanation for the unexpected findings in the tension reduction condition. In this study the tension increase and tension decrease conditions differed in the intensity of stimulation that was provided to the infant. Infants receiving tension increase were administered a larger quantity of stimulation compared to infants in the tension decrease condition. This difference favoring the tension increase condition may have resulted in a greater effect on their emotions. 
A third relevant factor in accounting for the unsuccessful consequences of the tension reduction condition is the lack of a deictic function for tactile stimulation; that is, tactile stimulation lacks referential specificity. This is in contrast to the facial modality in which a signaler of a facial display can gaze at a particular referent, thereby communicating to the other about a specific environmental event. In short, a deictic function may need to be added to the tactile modality. This may be accomplished in a number of ways, one of which is by orienting one's attention toward the event to which the touch manipulation refers. If this is so, we believe that a deictic function inadvertently was created for the tension increase condition but not for tension decrease. More specifically, the tension increase condition very likely resulted in the infants "better keeping an eye" on the objects than those in the tension decrease condition. Thus, one may not necessarily expect infants in the latter condition to regulate their behaviors to the objects per se.

There is another possible explanation for the unpredicted results, but it is one that we do not favor. It is possible that the specific context in which the tactile array was applied was unnatural or strange to the infant. We do not favor this explanation because the infants seemed to be relatively content while on their mothers' laps, at least for the 20 to $30 \mathrm{sec}$ after receiving the tactile manipulation. However, we can not rule out this explanation completely.

A final explanation concerns the different functional consequences of positive and negative emotion. As Cacioppo and Gardner (1999) pointed out, negative emotions often serve to block action tendencies, whereas positive emotions often serve to maintain (not necessarily increase) them. Most infants in this study had either a neutral or an approach action tendency as the objects were presented to them. If, as Cacioppo and Gardner argued, negative emotions are more effective in blocking actions than positive ones are in increasing them, it should be no surprise to discover strong effects in inhibiting action in the tension increase condition and a similarity between the control condition and a tension decrease condition that does not potentiate action tendencies. This may explain other studies of emotion in which negative emotional displays regulate infants' behavior much more than do positive emotional displays (e.g., Mumme et al., 1996).

This study is only a first step in understanding the parameters and qualities of touch that elicit emotion in the infant. There are a number of steps that should be taken to understand the role of touch in the emotional life of the infant. First, positive touch and its role in the elicitation of emotion should be investigated. Second, different parameters of tactile stimulation and different tactile qualities should be systematically varied to investigate the effect of contact on the elicitation of emotion. Third, developmental questions relating to the tactile modality should be addressed. For instance, does the regulatory role of touch wane when infants begin to crawl and the distal channels of communication (the face and voice) begin to play a more prominent role? In addition to developmental questions, the role that touch plays in the emotional lives of infants who are deaf or blind also merits attention 
and investigation. Only further research will illuminate the answers to these and other important questions.

\section{ACKNOWLEDGMENTS}

This research was supported by a NRSA fellowship from the National Institutes of Mental Health (MH-12320) to Matthew J. Hertenstein and by grants from NICHD (HD-25066 and HD-36795), National Science Foundation (SBR-9816151), and the John D. and Catherine T. MacArthur Foundation to Joseph J. Campos.

Portions of this research were presented at the Biennial Meetings of the Society for Research in Child Development, April 1999.

\section{REFERENCES}

Barnett, K. (1972). A theoretical construct of the concepts of touch as they relate to nursing. Nursing Research, 21, 102-110.

Birns, B., Blank, M., \& Bridger, W. (1966). The effectiveness of various soothing techniques on human neonates. Psychosomatic Medicine, 28, 316-322.

Boccia, M., \& Campos, J. J. (1989). Maternal emotional signals, social referencing, and infants' reactions to strangers. New Directions for Child Development, 44, 25-49.

Cacioppo, J. T., \& Gardner, W. L. (1999). Emotions. Annual Review of Psychology, 50, 191-214.

Camras, L. A., \& Sachs, V. B. (1991). Social referencing and caretaker expressive behavior in a day care setting. Infant Behavior \& Development, 14, 27-36.

Clark-Carter, D. (1997). Doing quantitative psychological research: From design to report. East Sussex, England: Taylor \& Francis.

Feinman, S., \& Lewis, M. (1984). Social referencing at ten months: A second-order effect on infants' responses to strangers. Child Development, 54, 878-887.

Field, T. M. (1983). Discrimination and imitation of facial expressions by term and preterm neonates. Infant Behavior \& Development, 6, 485-489.

Field, T. M. (1998). Massage therapy effects. American Psychologist, 53, 1270-1281.

Geldard, F. A. (1960, May 27). Some neglected possibilities of communication. Science, 131, $1583-1588$.

Hatfield, E., Cacioppo, J. T., \& Rapson, R. L. (1994). Emotional contagion. Cambridge, England: Cambridge University Press.

Hertenstein, M. J. (in press). Touch: Its communicative functions in infancy. Human Development.

Hirshberg, L. M., \& Svejda, J. (1990). When infants look to their parents: Infants' social referencing of mothers compared to fathers. Child Development, 61, 1175-1186.

Keppel, G. (1991). Design and analysis: A researcher's handbook (3rd ed.). Englewood Cliffs, NJ: Prentice Hall.

Klinnert, M. D. (1984). The regulation of infant behavior by maternal facial expression. Infant Behavior \& Development, 7, 447-465.

Koester, L. S., Papoušek, H., \& Papoušek, M. (1989). Patterns of rhythmic stimulation by mothers with three-month-olds: A cross-modal comparison. International Journal of Behavioral Development, 12, 143-154. 
Korner, A. F., \& Thoman, E. B. (1972). The relative efficacy of contact and vestibular-proprioceptive stimulation in soothing neonates. Child Development, 43, 443-453.

Mumme, D. L., Fernald, A., \& Herrera, C. (1996). Infants' responses to facial and vocal emotional signals in a social referencing paradigm. Child Development, 67, 3219-3237.

Peláez-Nogueras, M., Field, T., Gewirtz, J. L., Cigales, M., Gonzalez, A., Sanchez, A., \& Richardson, S. C. (1997). The effects of systematic stroking versus tickling and poking on infant behavior. Journal of Applied Developmental Psychology, 18, 169-178.

Peláez-Nogueras, M., Gewirtz, J. L., Field, T., \& Cigales, M. (1996). Infants' preference for touch stimulation in face-to-face interactions. Journal of Applied Developmental Psychology, 17, 199-213.

Repacholi, B. M. (1998). Infants' use of attentional cues to identify the referent of another person's emotional expression. Developmental Psychology, 34, 1017-1025.

Stack, D. M., \& Muir, D. W. (1990). Tactile stimulation as a component of social interchange: New interpretations for the still-face effect. British Journal of Developmental Psychology, 8, 131-145.

Stack, D. M., \& Muir, D. W. (1992). Adult tactile stimulation during face-to-face interactions modulates five-month-olds' affect and attention. Child Development, 63, 1509-1525.

Svejda, M. J. (1981). The development of infant sensitivity to affective messages in the mother's voice. Unpublished doctoral dissertation, University of Denver, Denver, CO.

Tomkins, S. S. (1982). Affect, imagery, consciousness (Vol. 3). New York: Springer.

Tronick, E. Z. (1995). Touch in mother-infant interaction. In T. M. Field (Ed.), Touch in early development (pp. 53-65). Mahwah, NJ: Lawrence Erlbaum Associates, Inc.

Tronick, E. Z., Als, H., Adamson, L., Wise, S., \& Brazelton, T. B. (1978). The infant's response to entrapment between contradictory messages in face-to-face interaction. Journal of American Academy of Child Psychiatry, 17, 1-13. 